\title{
Does Systemic Arterial Hypertension Change the Function of the Stomatognathic System?
}

João Batista Mattos Lellis ${ }^{1}$, Tânia de Freitas Borges², Richard Honorato de Oliveira', Marcelo Palinkas ${ }^{1,3}$, Jaime Eduardo Cecilio Hallak $^{3,4}$, Selma Siéssere ${ }^{1,3}$, Simone Cecilio Hallak Regalo ${ }^{1,3}$ ${ }^{1}$ Department of Basic and Oral Biology, School of Dentistry of Ribeirão Preto, University of São Paulo, São Paulo, Brazil;

${ }^{2}$ Department of Dental Prosthesis, Technical School of Health, Federal University of Uberlândia, Uberlândia, Brazil;

${ }^{3}$ National Institute and Technology - Translational Medicine (INCT.TM), São Paulo, Brazil;

${ }^{4}$ Department of Neuroscience and Behavioral Sciences, Faculty of Medicine of Ribeirão Preto, University of São Paulo, São Paulo, Brazil

Received October 10, 2020; Accepted August 3, 2021.

Key words: Hypertension - Masticatory muscle - Masticatory efficiency Bite force - Thickness

Abstract: The aim of this study was to evaluate the stomatognathic system of individuals with controlled systemic hypertension through comparison with a disease-free control group. Seventy individuals ( 44 female and 26 male) were divided into two groups: a controlled systemic hypertension $(n=35)$ and a disease-free control $(n=35)$. The individuals were evaluated on the basis of masticatory cycle efficiency of the value of the ensemble-averaged integrated linear envelope to the electromyographic signal of the masseter and temporalis muscles in the habitual (peanuts and raisins) and non-habitual chewing (Parafilm M); molar bite force (right and left) and ultrasound images from the bilateral masseter and temporal muscles at rest and maximum voluntary contraction. The data obtained were tabulated and submitted to statistical analysis $(p<0.05)$. There was a significant difference between

This study was supported by the Fundação de Amparo a Pesquisa do Estado de São Paulo (FAPESP, Brazil), and National Institute and Technology - Translational Medicine (INCT.TM), São Paulo.

Mailing Address: Prof. Marcelo Palinkas, PhD., School of Dentistry of Ribeirão Preto, University of São Paulo, Avenida do Café s/n, Bairro Monte Alegre CEP 14040-904, Ribeirão Preto, São Paulo, Brazil; e-mail: palinkas@usp.br 
groups in the habitual (peanuts and raisins) and non-habitual (Parafilm M) chewing with reduced muscle activity to controlled systemic hypertension group. Muscle thickness occurred significant difference between groups at rest and maximum voluntary contraction of the temporalis muscles. There was no significant difference between groups in maximum molar bite force. The present study findings indicate that the controlled systemic hypertension promotes functional changes of the masticatory system, especially with respect to its masticatory efficiency and muscle thickness.

\section{Introduction}

Diseases such as systemic arterial hypertension are associated with vascular changes mediated by reactive oxygen species that promote changes in vascular tonus, remodelling, and inflammation (Vaka et al., 2018; Xu et al., 2020; Yang et al., 2020). In addition to changes to the vascular system, structural changes of organs such as the kidneys, lungs and heart are also directly related to increased generation of reactive oxygen species in hypertension and cardiac hypertrophy (Garg et al., 2020).

The prevalence of systemic arterial hypertension in the world's population is $26 \%$ in adults, and it is believed to reach $40 \%$ in certain regions. It is estimated that this percentage will further increase by 2025 to reach 29\% (Kearney et al., 2005; Lacruz et al., 2015).

In the early stages of systemic arterial hypertension, functional factors predominate in elevating peripheral resistance; in chronic hypertension, structural factors such as vascular remodelling and hypertrophy contribute (Lewington et al., 2002). Muscle function in hypertensive individuals may be affected due to the increase in peripheral vascular resistance and inadequate regulation of oxygen supply to the skeletal muscle (Nyberg et al., 2015). Whereas the inducing oxidative stress in muscle during hypertension brings an increase of angiotensin II, muscle contraction stimulates thin fibers muscle afferents and evokes reflex sympathetic excitation (Koba et al., 2013).

The problems arising in the presence of systemic arterial hypertension involve the striated skeletal muscle system, with impairment of muscle function. Therefore, changes in the activity of the stomatognathic system may also occur. However, no work on systemic arterial hypertension has been found relating to hypertension and mastication muscles. This fact highlights the need to analyse the relationship between the stomatognathic system and chronic degenerative diseases. This study is aimed at the analysis of chewing cycles, muscle thickness, and bite force in individuals with hypertension.

The main hypothesis of the study was that systemic arterial hypertension disease promoted functional changes in the stomatognathic system. This research guides and directs health professionals to perform more cautious oral rehabilitation treatments with the function of promoting clinical success with better quality of life for patients with arterial hypertension. 


\section{Material and Methods}

Study design and sample

This observational study was approved by the ethics committee (process \# 2011.1.1162.58.9). The individuals were informed about the objectives and stages of the study and gave written informed consent.

The post hoc sample size was calculated considering a level of $\alpha=0.05$, a power of $100 \%$ for the main outcome Parafilm M chewing of the right master muscle (mean [standard deviation]: medically controlled hypertension group, 1.20 [0.13] and control group, 1.58 [0.11]) and effect size of 3.15. The minimal sample size obtained was 70 volunteers (35 for each group). The sample size was calculated with the G*Power 3.1.9.2 software.

From an initial cohort of 60 individuals medically controlled hypertension, both genders, with complete teeth and properly rehabilitated through fixed prosthesis of up to one element, 35 were selected for medically controlled hypertension group (mean [standard deviation], 49.2 [1.6] years) on the basis of the eligibility screening criteria.

Sample selection and criteria for inclusion and exclusion were determined by clinical examination and medical history. As inclusion criteria for World Health Organization, individuals should have medical diagnosis of hypertension, were treated, and controlled, and already make use of antihypertensive drugs for at least one year. All individuals should present at least 24 teeth, with at least a molar occlusion in each dental hemi-arch. Individuals with disorders affecting craniofacial growth, a history of epileptic seizures, with removable dental prostheses, and/or using medication or undergoing treatment that could directly or indirectly interfere with muscle activity (antihistamines, sedatives, homeopathy or other depressants of the central nervous system), presence of parafunctional habits, and possible symptoms of temporomandibular disorders (Research Diagnostic Criteria for Temporomandibular Disorders), were excluded from participation in the study.

The disease-free control group (mean [standard deviation], 49.1 [1.6] years) was comprised of individuals who were age, weight, and height matched with the individuals in medically controlled hypertension group (Table 1). Each group had 22 women and 13 men.

\section{Table 1 - Mean, standard error mean ( \pm ) and statistical significance $(p<0.05)$ of demographic data in hypertensive and disease-free control groups}

\begin{tabular}{lccc}
\hline Groups & Age & Weight & Height \\
\hline Hypertensive & $49.2 \pm 1.6$ & $77.06 \pm 2.78$ & $1.65 \pm 0.01$ \\
Control & $49.1 \pm 1.6$ & $72.81 \pm 1.77$ & $1.68 \pm 0.01$ \\
P-value & 0.20 & 0.21 & 0.96 \\
\hline
\end{tabular}


The tests applied in this study were performed at a single time by one calibrated investigator who participated in full in the collection of all data.

\section{Masticatory efficiency}

The electromyographic signals of the masticatory cycles were collected using the Myosystem BR-1 portable electromyograph (Data Hominis, Uberlandia, Minas Gerais, Brazil), with analog bandpass filters for a cut-off frequency of 10-1,000 Hz, scanning for sample frequency of $4 \mathrm{kHz}$, and 12-bit resolution. Silver/silver chloride bipolar surface electrodes (Data Hominis Ltd., Model DHT-EASD) with diameter and inter-electrode distance of $10 \mathrm{~mm}$ were used. The maximum voluntary contraction manoeuvre was performed for the correct positioning of the electrodes (Hermens et al., 2000).

During the collection of electromyographic data, the individuals remained seated upright, with the soles of their feet resting on the ground, their arms resting on their legs, and head erect with the plane of the head parallel to the ground.

In the analysis of the muscle activity during chewing, the dynamics of habitual and non-habitual chewing was determined using the linear envelopment of the electromyographic signals of the masseter and temporalis muscles (Siéssere et al., 2009; da Silva et al., 2019). Non-habitual chewing is a movement with dynamic records and short excursion during mouth opening (articulator type), which are required to reduce the effects of the change of length and muscle (De Luca, 1997).

The electromyographic signals were obtained during the habitual chewing of $5 \mathrm{~g}$ of peanuts (hard food) and $5 \mathrm{~g}$ of raisins (soft food). Non-habitual chewing was recorded with Parafilm $M$. Soft and hard food were selected from the same batch, separated in units, and stored in a plastic recipient. This material was kept in a cool and airy place.

At the beginning of the masticatory process, the initial cycles showed a variation in the pattern of the mandibular movement. Therefore, to calculate the results obtained from the integral of the linear envelope of the masticatory cycles, the initial masticatory cycles were eliminated while the central cycles of the electromyographic were maintained. Three initial masticatory cycles were excluded since, in the initial phase of the masticatory process, the first cycles vary considerably during mandibular movement (Palinkas et al., 2019; Righetti et al., 2020).

\section{Muscle thickness}

The portable ultrasound device Titan (SonoSite Inc., Bothell, WA, USA) was used for analysis of muscle thickness during the clinical conditions of at rest and dental clenching in maximum voluntary contraction. During collection, the linear transducer was placed transversely in the direction of the fibers of the belly of the masseter muscle, approximately $1.5 \mathrm{~cm}$ to $2.0 \mathrm{~cm}$ above the angle of the mandible toward the zygomatic arch. For the temporal muscle, the linear transducer was positioned in the region of the temporal fossa, about $1.0 \mathrm{~cm}$ to $1.5 \mathrm{~cm}$ behind and above the lateral 
palpebral commissures on both sides. Three measurements were performed on each muscle, separately (right masseter, left masseter, right temporal and left temporal), with the mean value being considered the muscle thickness (Palinkas et al., 2010; Donizetti et al., 2019).

Bite force

To obtain bite force, an IDDK (Kratos, Cotia, São Paulo, Brazil) model digital dynamometer was used, with a capacity of up to $1,000 \mathrm{~N}$ force, adapted to the oral cavity. The operator can choose the scale to be in Newtons. The measures were taken in the first permanent molars (right and left). Bite force was recorded in newton, each subject was asked to bite the device three times with maximum effort, with a 2-min rest between trials. The highest value among three trials was considered the subject's (Palinkas et al., 2010; Verma et al., 2017).

Statistical analysis

After obtaining the masticatory efficiency, muscle thickness and bite force data, a normality test was run, and the data were considered normally distributed. The data were statistically analysed (Statistical Package for the Social Sciences Version 22.0 for Windows, IBM Inc., Chicago, IL, USA). The results were obtained using descriptive analysis for each variable. The values were compared using the student's $t$-test for independent samples, considering a confidence level of $95 \%(p<0.05)$.

\section{Results}

Table 2 shows the data of muscle activity during habitual (peanuts and raisins) and non-habitual chewing (Parafilm M) of masseter and temporalis muscles, thar were

Table 2 - Mean, standard error mean ( \pm ) and statistical significance $(p<0.05)^{*}$ of integral of linear envelope in chewing, for each muscle evaluated, in the hypertensive group (HG) and disease-free control (CG)

\begin{tabular}{lcccc}
\hline Chewing & Muscles & HG & CG & P-value \\
\hline \multirow{3}{*}{ Parafilm M } & right masseter & $1.20 \pm 0.13$ & $1.58 \pm 0.11$ & $0.03^{*}$ \\
& left masseter & $1.03 \pm 0.12$ & $1.66 \pm 0.13$ & $0.01^{*}$ \\
& right temporal & $0.94 \pm 0.09$ & $1.38 \pm 0.09$ & $0.00^{*}$ \\
& left temporal & $1.11 \pm 0.12$ & $1.42 \pm 0.09$ & $0.05^{*}$ \\
\hline \multirow{3}{*}{ Raisins } & right masseter & $0.93 \pm 0.10$ & $1.47 \pm 0.32$ & $0.00^{*}$ \\
& left masseter & $0.88 \pm 0.08$ & $1.21 \pm 0.10$ & $0.00^{*}$ \\
& right temporal & $0.88 \pm 0.09$ & $1.06 \pm 0.08$ & $0.05^{*}$ \\
& left temporal & $0.90 \pm 0.09$ & $1.18 \pm 0.08$ & $0.01^{*}$ \\
\hline \multirow{3}{*}{ Peanuts } & right masseter & $1.25 \pm 0.09$ & $1.71 \pm 0.12$ & 0.11 \\
& left masseter & $1.11 \pm 0.09$ & $1.68 \pm 0.10$ & $0.01^{*}$ \\
& right temporal & $1.19 \pm 0.12$ & $1.47 \pm 0.09$ & 0.16 \\
& left temporal & $1.23 \pm 0.13$ & $1.82 \pm 0.14$ & $0.03^{*}$ \\
\hline
\end{tabular}


Table 3 - Mean, standard error mean ( \pm ), and statistical significance $(p<0.05) *$ of muscle thickness $(\mathrm{mm})$ in the clinical condition of at rest and dental clenching, in the hypertensive group (HG) and disease-free control (CG)

\begin{tabular}{llccc}
\hline Clinical conditions and muscles & HG & CG & P-value \\
\hline \multirow{3}{*}{ Rest } & Right masseter & $1.06 \pm 0.03$ & $1.00 \pm 0.02$ & 0.21 \\
& Left masseter & $1.05 \pm 0.03$ & $1.01 \pm 0.02$ & 0.37 \\
& Right temporal & $0.40 \pm 0.01$ & $0.64 \pm 0.02$ & $0.00^{*}$ \\
& Left temporal & $0.39 \pm 0.02$ & $0.63 \pm 0.02$ & $0.00^{*}$ \\
\multirow{3}{*}{ Dental } & Right masseter & $1.34 \pm 0.04$ & $1.27 \pm 0.03$ & 0.25 \\
clenching & Left masseter & $1.33 \pm 0.04$ & $1.28 \pm 0.03$ & 0.40 \\
& Right temporal & $0.51 \pm 0.02$ & $0.72 \pm 0.02$ & $0.00^{*}$ \\
& Left temporal & $0.50 \pm 0.02$ & $0.71 \pm 0.02$ & $0.00^{*}$ \\
\hline
\end{tabular}

Table 4 - Mean, standard error mean ( \pm ) and statistical significance $(p<0.05)^{*}$ of the bite force of the right and left side molar regions in the hypertensive group (HG) and disease-free control (CG)

\begin{tabular}{lccc}
\hline Force & HG & CG & P-value \\
\hline Right molar & $303.51 \pm 28.43$ & $260.46 \pm 26.77$ & 0.28 \\
Left molar & $321.85 \pm 27.94$ & $280.27 \pm 33.04$ & 0.33 \\
\hline
\end{tabular}

analysed using the integral of linear envelope. The muscle activity with chewing decreased for hypertension group in hard food and soft food, with a significant difference $(p<0.05)$; with exception for the masseter and temporal right muscles during chewing with peanuts where no difference was found.

The average thickness of the muscle at rest and at the maximum voluntary contraction in temporalis muscles (left and right) were lower in the hypertension group $(p<0.05)$ (Table 3).

The maximum molar bite force values as shown in Table 4. There were no significant differences $(p<0.05)$ between both groups.

\section{Discussion}

The purpose of this study was to determine the morphological and functional changes in individuals with medicated and controlled hypertension by using methodologies for measuring masticatory efficiency, muscle thickness and molar bite force.

The muscle activity during chewing showed a decrease of electromyographic activity in all muscles analysed for the hypertensive group. Chewing is a complex and dynamic process. The basic motor pattern is modified by a sensory feedback with 
influences of central and peripheral afferents that control the chewing muscles during the most critical phases of mastication (Bosman et al., 2004).

The non-habitual mastication of Parafilm $M$ is a standardized method, eliminating the interfering factors that act during the masticatory process, such as preferred side of mastication, swallowing between the chewing cycles, frequency of mastication and the texture of the food. In this study, in the clinical condition of non-habitual mastication with Parafilm M, there was a decrease of electromyographic activity in all muscles analysed for the hypertensive group. During non-habitual mastication of Parafilm M, moderate activity without the force is required from the muscles, so we can consider that the dentate individuals without disorders really are those with the more balanced musculature of the stomatognathic system (Siéssere et al., 2009).

The habitual, natural, non-oriented mastication pattern consists of alternating the side of work and is responsible for the fragmentation of food by the teeth by means of cyclic mandibular movements of opening and closing, called chewing cycles. Alterations caused by occlusion of the joints or muscles of mastication affect chewing efficiency (Kim et al., 1997). In mastication of soft (raisins) or hard (peanuts) foods, it was also found that the electromyographic values of the chewing cycles were lower for the hypertensive group.

The contraction of masticatory muscles is dynamic and successive during mastication. A critical component in regulating skeletal muscle contractility is the release of $\mathrm{Ca}^{2+}$ via ryanodine receptor (RyR) $\mathrm{Ca}^{2+}$ release channels in the sarcoplasmic reticulum (Andersson and Marks, 2010). There are indications that beta-blockers affect the skeletal muscle in therapeutic dosages (Finsterer and Gelpi, 2006).

Calcium channel blockers are commonly used in some cardiovascular disorders. These drugs can act at neuromuscular transmission, at both pre- and postsynaptic levels and may produce neuromuscular dysfunction. The calcium channel blockers, as verapamil and amlodipine, can impair neuromuscular transmission in individuals without neuromuscular disease, which may cause misinterpretation of single fiber electromyography studies carried out to investigate neuromuscular junction disorders (Ozkul, 2007; Seydi et al., 2020). In our study, 17\% and 53\% of hypertensive individuals make use calcium channel blockers and diuretics, respectively.

The efficacy and low cost of thiazide and thiazide-like diuretics become the most used as a potent medication option for many patients with hypertension (Duarte and Cooper-DeHoff, 2010). In our study, almost $100 \%$ of diuretic users has been treated with thiazide.

The members of the diuretic class of drugs vary greatly in structure, physicochemical properties and site and mechanism of action. Diuretics are drugs that increase the rate of urine flow and sodium $\left(\mathrm{Na}^{+}\right)$excretion to adjust the volume and composition of body fluids (Cadwallader et al., 2010). 
During excitation, muscle cells gain $\mathrm{Na}^{+}$and lose $\mathrm{K}^{+}$, leading to a rise in extracellular $\mathrm{K}^{+}\left(\left[\mathrm{K}^{+}\right] \mathrm{o}\right)$, depolarization, and loss of excitability. Recent studies support the idea that these events are important causes of muscle fatigue and that full use of the $\mathrm{Na}^{+}, \mathrm{K}^{+}$-ATPase (adenosine triphosphatase) (also known as the $\mathrm{Na}^{+}$, $\mathrm{K}^{+}$pump) is often essential for adequate clearance of extracellular $\mathrm{K}^{+}$(Clausen, 2013).

In addition to the possible adverse effects of anti-hypertensive drugs in skeletal muscle, several changes in peripheral resistancy associated with hypertension may involve also the microvascular network (Vicaut, 2003). The supply of the tissues with oxygen, nutrients, and metabolites occurs almost exclusively in the microcirculation (Jung et al., 2013). In hypertension, the endothelial function and regulation of vascular tone is impaired with consequent increases in peripheral vascular resistance and inadequate regulation of oxygen supply to the skeletal muscle, which can affect muscle function (Nyberg et al., 2015).

The fact that the individuals with hypertension have lower electromyographic activity during mastication in comparison with the activity developed by the control individuals in the same clinical conditions, could indicate dysfunction of stomatognathic system caused by hypertension or by the anti-hypertension medication.

When muscle thickness was compared between the hypertensive and control groups, it was found that the thicknesses were quite similar for the masseter muscles, but for the temporal muscles, the values were quite different, being lower in the hypertensive group both at rest and at maximum voluntary contraction and these results are concurrent with international research (Bertram et al., 2003).

The morphological and functional characteristics of the muscles are different, where the masseter is a muscle potent with the function of force, which carries and maintains the bones, protecting and leading the power of the movement, and elevating the mandible anti-gravity during the various functions of the stomatognathic system. Temporal muscle function is more related to speed, being the first to contract when the mandible is closed, and considered to be a mandible positioner, adjusting the direction of movement, and acting as synchronizer of movements (Koolstra, 2002). The other researches show that muscles with a high proportion of fast fibers have a higher resistance than muscles rich in slow fibers and suggest that the type of fiber in skeletal muscle might be of importance for the development of the hypertensive disease (Juhlin-Dannfelt et al., 1979).

Although the muscle activity during chewing showed a decrease of electromyographic activity in all muscles analysed and the lower thickness for the temporal muscle for the hypertensive group, the similar bite force was verified between hypertensive and disease-free control group. The fact of bite force was not affected by the lower thickness of the temporal muscle in the hypertensive group individuals may be due to the ability of muscle fibers to adapt to new functional requirements to optimize their contractile function (Korfage et al., 2005). 
In statistical analysis comparison between hypertensive and disease-free control groups, it was found that some factors such as body mass index, weight, age and height showed not significant differences within the group, only body mass index was a statistically significant factor among the groups.

It is known that the most hypertensive individuals are overweight, and one of the main recommendations for their treatment is to lose the excess weight. Further, clinical studies are necessary in order to define more precisely the risk factors for hypertensive diseases and the degree to which the stomatognathic system of hypertensive individuals is compromised, particularly assessing the type and duration of medication use, as well as time of hypertensive disease. Factors such as age, gender and body mass index should also be included in future longitudinal studies.

The present study was limited due to the group of patients with systemic arterial hypertension is very small, requiring further studies in this area of health.

\section{Conclusion}

The authors suggest that systemic arterial hypertension promotes functional changes of the masticatory system, especially with respect to its muscle activity during chewing and thickness for the temporal muscle, but without interference on the molar bite force.

\section{References}

Andersson, D. C., Marks, A. R. (2010) Fixing ryanodine receptor Ca leak - A novel therapeutic strategy for contractile failure in heart and skeletal muscle. Drug Discov. Today Dis. Mech. 7, e151-e157.

Bertram, S., Brandlmaier, I., Rudisch, A., Bodner, G., Emshoff, R. (2003) Cross-sectional characteristics of the masseter muscle: an ultrasonographic study. Int. J. Oral Maxillofac. Surg. 32, 64-68.

Bosman, F., Van der Bilt, A., Abbink, J. H., Van der Glas, H. W. (2004) Neuromuscular control mechanisms in human mastication. J. Texture Stud. 35, 201-221.

Cadwallader, A. B., de la Torre, X., Tieri, A., Botrè, F. (2010) The abuse of diuretics as performanceenhancing drugs and masking agents in sport doping: Pharmacology, toxicology and analysis. Br. J. Pharmacol. 161, 1-16.

Clausen, T. (2013) Quantification of $\mathrm{Na}^{+}, \mathrm{K}^{+}$pumps and their transport rate in skeletal muscle: Functional significance. J. Gen. Physiol. 142, 327-345.

da Silva, N., Verri, E., Palinkas, M., Hallak, J., Regalo, S., Siéssere, S. (2019) Impact of Parkinson's disease on the efficiency of masticatory cycles: Electromyographic analysis. Med. Oral Patol. Oral Cir. Bucal 24, e314-e318.

De Luca, C. J. (1997) The use of surface electromyography in biomechanics. J. Appl. Biomech. 13, $135-163$.

Donizetti Verri, E., da Silva, G. P., Marianetti Fioco, E., Soares da Silva, N., Valin Fabrin, S. C., Augusto Bueno Zanella, C., Roberta Garrefa, C., Faria Júnior, M., Siéssere, S., Hallak, J. E. C., Palinkas, M., Chaves, T. C., Regalo, S. C. H. (2019) Effects of Parkinson's disease on molar bite force, electromyographic activity and muscle thickness of the masseter, temporal and sternocleidomastoid muscles: a case-control study. J. Oral Rehabil. 46, 912-919.

Duarte, J. D., Cooper-DeHoff, R. M. (2010) Mechanisms for blood pressure lowering and metabolic effects of thiazide and thiazide-like diuretics. Expert Rev. Cardiovasc. Ther. 8, 793-802.

Finsterer, J., Gelpi, E. (2006) Mitochondrial disorder aggravated by propranolol. South. Med. J. 99, $768-771$. 
Garg, A., Foinquinos, A., Jung, M., Janssen-Peters, H., Biss, S., Bauersachs, J., Gupta, S. K., Thum, T. (2020) MiRNA-181a is a novel regulator of aldosterone-mineralocorticoid receptor-mediated cardiac remodelling. Eur. J. Heart Fail. 22, 1366-1377.

Hermens, H. J., Freriks, B., Disselhorst-Klug, C., Rau, G. (2000) Development of recommendations for SEMG sensors and sensor placement procedures. J. Electromyogr. Kinesiol. 10, 361-374.

Juhlin-Dannfelt, A., Frisk-Holmberg, M., Karlsson, J., Tesch, P. (1979) Central and peripheral circulation in relation to muscle-fibre composition in normo- and hyper-tensive man. Clin. Sci. (Lond.) 56, 335-340.

Jung, F., Pindur, G., Ohlmann, P., Spitzer, G., Sternitzky, R., Franke, R. P., Leithäuser, B., Wolf, S., Park, J. W. (2013) Microcirculation in hypertensive patients. Biorheology 50, 241-255.

Kearney, P. M., Whelton, M., Reynolds, K., Muntner, P., Whelton, P. K., He, J. (2005) Global burden of hypertension: Analysis of worldwide data. Lancet 365, 217-223.

Kim, Y. K., Lee, S. W., Chung, S. C., Kho, H. S. (1997) Comparison of muscle activity and occlusal contacts during maximum and habitual clenching in varied chair positions. J. Oral Rehabil. 24, 237-239.

Koba, S., Watanabe, R., Kano, N., Watanabe, T. (2013) Oxidative stress exaggerates skeletal muscle contraction-evoked reflex sympathoexcitation in rats with hypertension induced by angiotensin II. Am. J. Physiol. Heart Circ. Physiol. 304, H142-H153.

Koolstra, J. H. (2002) Dynamics of the human masticatory system. Crit. Rev. Oral Biol. Med. 13, 366-376.

Korfage, J. A., Koolstra, J. H., Langenbach, G. E., van Eijden, T. M. (2005) Fiber-type composition of the human jaw muscles - (Part 1) Origin and functional significance of fiber-type diversity. J. Dent. Res. 84, 774-783.

Lacruz, M. E., Kluttig, A., Hartwig, S., Löer, M., Tiller, D., Greiser, K. H., Werdan, K., Haerting, J. (2015) Prevalence and incidence of hypertension in the general adult population: Results of the CARLA-cohort study. Medicine (Baltimore) 94, e952.

Lewington, S., Clarke, R., Qizilbash, N., Peto, R., Collins, R.; Prospective Studies Collaboration (2002) Agespecific relevance of usual blood pressure to vascular mortality: A meta-analysis of individual data for one million adults in 61 prospective studies. Lancet 360, 1903-1913.

Nyberg, M., Gliemann, L., Hellsten, Y. (2015) Vascular function in health, hypertension, and diabetes: Effect of physical activity on skeletal muscle microcirculation. Scand. J. Med. Sci. Sports 4, 60-73.

Ozkul, Y. (2007) Influence of calcium channel blocker drugs in neuromuscular transmission. Clin. Neurophysiol. 118, 2005-2008.

Palinkas, M., Nassar, M. S., Cecílio, F. A., Siéssere, S., Semprini, M., Machado-de-Sousa, J. P., Hallak, J. E., Regalo, S. C. (2010) Age and gender influence on maximum bite force and masticatory muscles thickness. Arch. Oral Biol. 55, 797-802.

Palinkas, M., Seidel, L., Hirono, T., Bataglion, C., De Luca Canto, G., de Mello, E., Gonçalves, L., Siéssere, S., Regalo, S. (2019) Influence of sleep bruxism severity on masticatory efficiency: Electromyographic analysis. Arch. Ital. Biol. 157, 59-65.

Righetti, M. A., Taube, O. L. S., Palinkas, M., Gonçalves, L. M. N., Esposto, D. S., de Mello, E. C., Regalo, I. H., Regalo, S. C. H., Siéssere, S. (2020) Osteoarthrosis: Analyze of the molar bite force, thickness and masticatory efficiency. Prague Med. Rep. 121, 87-95.

Seydi, E., Tabbati, Y., Pourahmad, J. (2020) Toxicity of atenolol and propranolol on rat heart mitochondria. Drug Res. (Stuttg.) 70, 151-157.

Siéssere, S., Lima, N., Semprini, M., de Sousa, L. G., Issa, J., Monteiro, S., Regalo, S. (2009) Masticatory process in individuals with maxillary and mandibular osteoporosis: Electromyographic analysis. Osteoporos. Int. 20, 1847-1851.

Vaka, V. R., McMaster, K. M., Cunningham, M. W. Jr., Ibrahim, T., Hazlewood, R., Usry, N., Cornelius, D. C., Amaral, L. M., LaMarca, B. (2018) Role of mitochondrial dysfunction and reactive oxygen species in 
mediating hypertension in the reduced uterine perfusion pressure rat model of preeclampsia. Hypertension 72, 703-711.

Verma, T. P., Kumathalli, K. I., Jain, V., Kumar, R. (2017) Bite force recording devices - A review. J. Clin. Diagn. Res. 11, ZE01-ZE05.

Vicaut, E. (2003) Hypertension and the microcirculation. Arch. Mal. Coeur Vaiss. 96, 893-903.

Xu, N., Jiang, S., Persson, P. B., Persson, E. A., Lai, E. Y., Patzak, A. (2020) Reactive oxygen species in renal vascular function. Acta Physiol. (Oxf.) 229, e13477.

Yang, G., Tan, Z., Zhou, L., Yang, M., Peng, L., Liu, J., Cai, J., Yang, R., Han, J., Huang, Y., He, S. (2020) Effects of angiotensin II receptor blockers and ACE (angiotensin-converting enzyme) inhibitors on virus infection, inflammatory status, and clinical outcomes in patients with COVID-19 and hypertension: A single-center retrospective study. Hypertension 76, 51-58. 